\title{
Bedaquiline-based treatment regimen for multidrug-resistant tuberculosis
}

\author{
To the Editor:
}

Treatment outcomes for multidrug-resistant (MDR) tuberculosis (TB) are poor with only approximately $50 \%$ of patients attaining treatment success [1]. An important driver of treatment failure in many settings is the unavailability of effective drugs for MDR- and extensively drug-resistant (XDR)-TB.

Until recently, the drugs used for TB treatment were largely developed decades prior or were repurposed for the treatment of MDR-TB [2]. In 2014, the European Medicines Agency approved bedaquiline for the treatment of MDR-TB making it the first drug in decades approved for this indication. Clinical trials have shown that bedaquiline is very effective leading to a shorter time to culture conversion in comparison to placebo $[3,4]$. A treatment regimen containing bedaquiline led to a 6 -month culture conversion in $79.5 \%$ of patients in a phase 2 clinical trial [5]. Due to an unexplained increased mortality in clinical trials and the potential for QT-interval prolongation, the World Health Organization advises caution for prescribers [6]. Nevertheless, in rare reports outside the clinical trial setting, bedaquiline was safe and effective leading to culture conversion in the majority of patients [7-10].

To add to the sparse knowledge, we aim to describe treatment responses of bedaquiline-based regimen use in patients with MDR/XDR-TB at the clinical referral centre in Germany.

We conducted a review of medical records of patients with an MDR/XDR-TB treated with bedaquiline between 2011 and 2016 at the Research Center Borstel, a referral centre for patients with MDR-TB from all regions of Germany.

MDR-TB was defined as the presence of isoniazid and rifampicin resistance, while XDR-TB was defined as MDR-TB plus additional resistance to fluoroquinolones and second-line injectable drugs (amikacin, kanamycin or capreomycin).

For primary detection, enrichment and drug susceptibility testing (DST) of streptomycin, isoniazid, rifampicin, ethambutol and pyrazinamide, the MGIT960 system (Becton Dickinson, Franklin Lakes, NJ, USA) was used. The second-line DST was performed as described previously [11]. The following drugs and concentrations (in $\mu \mathrm{g} \cdot \mathrm{mL}^{-1}$ ) were tested: isoniazid (0.1), rifampicin (1.0), rifabutin (0.25), amikacin (1.0), capreomycin (2.5), ofloxacin (2.0), levofloxacin (1.0), moxifloxacin (0.5), prothionamide (2.5), linezolid 1.0), ethambuthol (5.0), pyrazinamide (100.0) and $p$-aminosalicylic acid (4.0). Cycolserine was tested with the standard Löwenstein-Jensen proportion method at $40.0 \mu \mathrm{g} \cdot \mathrm{mL}^{-1}$.

Treatment was initiated on an individualised basis using molecular and phenotypic DST. Patients with MDR-TB received treatment as in-patients until culture conversion. As most of the patients are currently still under treatment, we used 6-month culture conversion rates as proxy microbiological outcomes to evaluate treatment response.

The study was approved by the ethical committee of the University of Lübeck, Germany (\#15-195A).

A total of 30 patients with MDR/XDR-TB were included. Median age was 29.5 (interquartile range (IQR) 23-39) years and 19 (63\%) were male. 27 (90\%) patients were born outside Germany and 21 (70\%) originated from countries from the former Soviet Union. Pulmonary TB was diagnosed in 29 (97\%) patients and one had pleural TB only. 17 (59\%) patients had received prior treatment for TB. Cavitary disease was present in $23(82 \%)$ patients and 17 (61\%) had bilateral lung involvement. 22 (73\%) patients

@ERSpublications

Bedaquiline-based therapy leads to high rates of culture conversion in the treatment of MDR-TB http://ow.ly/RGr230bqlVD

Cite this article as: Olaru ID, Heyckendorf J, Andres S, et al. Bedaquiline-based treatment regimen for multidrug-resistant tuberculosis. Eur Respir J 2017; 49: 1700742 [https://doi.org/10.1183/13993003.007422017]. 
were smear positive upon starting treatment. All patients had culture-confirmed MDR-TB and phenotypic DST results available.

Six (20\%) patients had MDR-TB only, while two (7\%) and seven (23\%) had additional resistance to second-line injectable drugs and fluoroquinolones. 15 (50\%) had XDR-TB and of those 13 (87\%) originated from countries of the former Soviet Union. Resistance to all first-line drugs was present in 25 (83\%) patients.

The median number of effective drugs used was six (IQR 5-7). The median time to bedaquiline initiation was 5.5 (IQR 0-41) days from the start of the MDR-TB therapy and 22 (73\%) patients were started on bedaquiline within the first month of treatment. All patients received at least 6 months of a bedaquiline-containing regimen.

In patients where bedaquiline was started within 1 month of MDR-TB treatment start, the median time to smear conversion was 52 (IQR 4-87) days, while median time to culture conversion was 49 (13-74) days. For two patients time to culture conversion could not be calculated due to lack of positive cultures from respiratory samples. Culture conversion was attained within 8 weeks of initiating MDR-TB treatment in 12 $(60 \%)$ patients and within 6 months in $20(100 \%)$ patients (figure 1). In patients with XDR-TB median time to culture conversion was 54 (IQR 46-87) days while in patients with non-XDR-TB culture conversion occurred after a median of 14 (IQR 4-59) days.

Adverse events were encountered in 23 (77\%) of patients, mostly associated with the other drugs in the regimen. Bedaquiline was safe and generally well tolerated and none of the patients required treatment discontinuation because of bedaquiline-related adverse events. Median number of electrocardiograms performed per month during in-hospital stay was 1.5 (IQR 0.8-2.7). Although QT-interval prolongation was observed in 20/21 (95\%) patients, no cardiac arrhythmias or deaths were recorded. One patient had a $\mathrm{QT}_{\mathrm{cB}}$ prolongation $\geqslant 60 \mathrm{~ms}$ and one patient had a prolongation exceeding $500 \mathrm{~ms}$ recorded on a single occasion.

We observed a very high and sustained rate of Mycobacterium tuberculosis culture conversion by 6 months of combination therapy with a bedaquiline-containing DST-individualised treatment regimen for patients with MDR-TB. Treatment with bedaquiline was not associated with adverse events requiring treatment discontinuation.

There are very few published studies on the effectiveness of bedaquiline treatment outside the clinical trial setting, especially from high-income countries. One study from France showed that MDR-TB patients treated with bedaquiline had a 6-month culture conversion rate of $97 \%$ with a median time to culture conversion of 85 days [7]. We found similar high rates of culture conversion after 6 months of therapy with a slightly shorter time to culture conversion perhaps due to the lower proportion of patients with smear positive disease and the higher number of active drugs associated within the regimen. $80 \%$ of patients from the French cohort had treatment success [12]. Another study from South-Africa reported that $76 \%$ of patients had culture conversion within the first 6 months [8]. A meta-analysis of 391 patients from five cohorts showed that almost $79.7 \%$ of patients treated with bedaquiline had culture conversion at the end of the initial 6 months of therapy and $69 \%$ had successful treatment [13].

Despite the very encouraging findings, bedaquiline resistance has been reported, supporting its rational use within an appropriate MDR-TB treatment regimen $[14,15]$.

FIGURE 1 Culture conversion in multidrug- and extensively drug-resistant tuberculosis patients treated with a bedaquiline-based regimen.

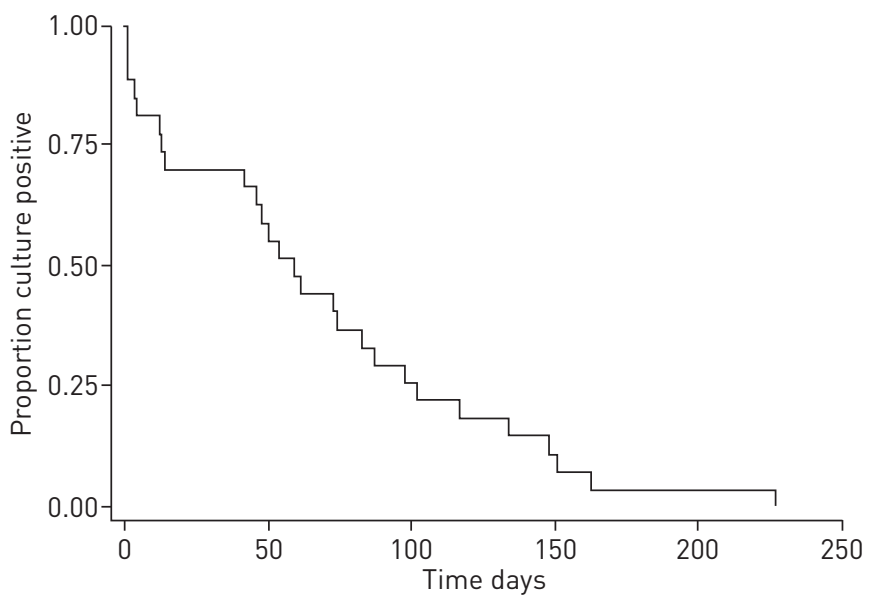


Although anticipated to become more available in low-resource settings, the widespread use of bedaquiline will still be hampered by its high costs. In Germany, the costs of treatment using a regimen including bedaquiline were estimated to be around $€ 85000$ per patient. However, adding bedaquiline to the background regimen would likely be a cost-effective intervention [16].

The study is limited by its retrospective study design, the number of patients included, and the inability to collect detailed data on adverse events. Due to the novelty of the drug data on patients treated with bedaquiline outside clinical trials is still scarce. Here we provide evidence for the effectiveness of bedaquiline-based regimens in clinical practice. Due to the recent approval of bedaquiline, most patients included in our study are currently still under MDR-TB treatment preventing us from evaluating treatment outcomes at the end of therapy. Two studies have shown that sputum culture conversion within the first 6 months can be used to predict treatment outcome $[17,18]$. Due to the novelty of the drug data on patients treated with bedaquiline outside the clinical trial setting is still scarce.

This study shows that treatment with bedaquiline in a low-burden high-income setting can lead to very high rates of culture conversion and potentially treatment success in patients with MDR/XDR-TB. Although the number of patients treated with bedaquiline is still small, the drug appears to be safe and well tolerated. Given these very encouraging results, bedaquiline should be available for all patients with MDR/XDR-TB for whom an effective treatment regimen cannot be offered otherwise.

Ioana D. Olaru $\mathbb{1}^{1,2}$, Jan Heyckendorf ${ }^{1,3}$, Sönke Andres ${ }^{4}$, Barbara Kalsdorf ${ }^{1,3}$ and Christoph Lange La,3,6 $^{1,3}$

${ }^{1}$ Division of Clinical Infectious Diseases, Research Center Borstel, Borstel, Germany. ${ }^{2}$ Dept of Microbiology, University Hospitals of Leicester, Leicester, UK. ${ }^{3}$ German Center for Infection Research (DZIF), Clinical Tuberculosis Unit, Borstel, Germany. ${ }^{4}$ Division of Mycobacteriology (National Tuberculosis Reference Laboratory), Research Center Borstel, Borstel, Germany. ${ }^{5}$ Dept of Medicine, Karolinska Institute, Stockholm, Sweden. ${ }^{6}$ Dept of Medicine, University of Namibia School of Medicine, Windhoek, Namibia.

Correspondence: Christoph Lange, Division of Clinical Infectious Diseases, Research Center Borstel, Parkallee 35, D23845 Borstel, Germany. E-mail: clange@fz-borstel.de

Received: April 102017 | Accepted after revision: April 292017

Conflict of interest: Disclosures can be found alongside this article at erj.ersjournals.com

\section{References}

1 World Health Organization. Global Tuberculosis Report 2016. Geneva, WHO, 2016.

2 Olaru ID, von Groote-Bidlingmaier F, Heyckendorf J, et al. Novel drugs against tuberculosis: a clinician's perspective. Eur Respir J 2015; 45: 1119-1131.

3 Diacon AH, Pym A, Grobusch M, et al. The diarylquinoline TMC207 for multidrug-resistant tuberculosis. $N$ Engl J Med 2009; 360: 2397-2405.

4 Diacon AH, Pym A, Grobusch MP, et al. Multidrug-resistant tuberculosis and culture conversion with bedaquiline. N Engl J Med 2014; 371: 723-732.

5 Pym AS, Diacon AH, Tang SJ, et al. Bedaquiline in the treatment of multidrug- and extensively drug-resistant tuberculosis. Eur Respir J 2016; 47: 564-574.

6 World Health Organization. Report of the Guideline Development Group Meeting on the use of bedaquiline in the treatment of multidrug-resistant tuberculosis. A review of available evidence (2016). Geneva, World Health Organization, 2017.

7 Guglielmetti L, Le Dû D, Jachym $\mathrm{M}$, et al. Compassionate use of bedaquiline for the treatment of multidrug-resistant and extensively drug-resistant tuberculosis: interim analysis of a French cohort. Clin Infect Dis 2015; 60: 188-194.

8 Ndjeka N, Conradie F, Schnippel K, et al. Treatment of drug-resistant tuberculosis with bedaquiline in a high HIV prevalence setting: an interim cohort analysis. Int J Tuberc Lung Dis 2015; 19: 979-985.

9 Skrahina A, Hurevich H, Falzon D, et al. Bedaquiline in the multidrug-resistant tuberculosis treatment: Belarus experience. Int J Mycobacteriol 2016; 5 Suppl 1: S62-SS3.

10 Udwadia ZF, Ganatra S, Mullerpattan JB. Compassionate use of bedaquiline in highly drug-resistant tuberculosis patients in Mumbai, India. Eur Respir J 2017; 49: 1601699.

11 Bemer P, Palicova F, Rüsch-Gerdes S, et al. Multicenter evaluation of fully automated BACTEC Mycobacteria Growth Indicator Tube 960 system for susceptibility testing of Mycobacterium tuberculosis. J Clin Microbiol 2002; 40: $150-154$.

12 Guglielmetti L, Jaspard M, Le Du D, et al. Long-term outcome and safety of prolonged bedaquiline treatment for multidrug-resistant tuberculosis. Eur Respir J 2017; 49: 1601799.

13 Mbuagbaw L. Review of available evidence on the use of bedaquiline for the treatment of multidrug-resistant tuberculosis: Data analysis report. Prepared for the World Health Organization, 2017. www.who.int/tb/ publications/2017/Appendix_GDGReport_Bedaquiline.pdf Date last updated: March 8, 2017.

14 Hoffmann H, Kohl TA, Hofmann-Thiel S, et al. Delamanid and bedaquiline resistance in Mycobacterium tuberculosis ancestral beijing genotype causing extensively drug-resistant tuberculosis in a tibetan refugee. Am J Respir Crit Care Med 2016; 193: 337-340.

15 Veziris N, Bernard C, Guglielmetti L, et al. Rapid emergence of Mycobacterium tuberculosis bedaquiline resistance: lessons to avoid repeating past errors. Eur Respir J 2017; 49: 1601719.

16 Wirth D, Dass R, Hettle R. Cost-effectiveness of adding novel or group 5 interventions to a background regimen for the treatment of multidrug-resistant tuberculosis in Germany. BMC Health Serv Res 2017; 17: 182. 
17 Kurbatova EV, Cegielski JP, Lienhardt C, et al. Sputum culture conversion as a prognostic marker for end-of-treatment outcome in patients with multidrug-resistant tuberculosis: a secondary analysis of data from two observational cohort studies. Lancet Respir Med 2016; 3: 201-209.

18 Günther G, Lange C, Alexandru S, et al. Treatment Outcomes in Multidrug-Resistant Tuberculosis. N Engl J Med 2016; 375: 1103-1105.

Copyright (CERS 2017 